\title{
Endoscopic retrograde cholangiopancreatography in octogenarians: A population-based study using the nationwide inpatient sample
}

Authors

Institutions
Clancy J. Clark' ${ }^{1}$ Adam $\mathrm{Coe}^{2}$, Nora F. Fino ${ }^{3}$, Rishi Pawa ${ }^{2}$

${ }^{1}$ Division of Surgical Oncology, Department of General Surgery, Wake Forest Baptist Health, Winston-Salem, North Carolina, United States

${ }^{2}$ Division of Gastroenterology, Department of Internal Medicine, Wake Forest Baptist Health, Winston-Salem, North Carolina, United States

${ }^{3}$ Division of Public Health Sciences, Wake Forest Baptist Health, Winston-Salem, North Carolina, United States submitted

19. November 2015

accepted after revision

7. March 2016

\section{Bibliography}

DOI http://dx.doi.org/

10.1055/s-0042-105432

Published online: 15.4.2016

Endoscopy International Open

2016; 04: E624-E630

(C) Georg Thieme Verlag KG

Stuttgart · New York

E-ISSN 2196-9736

\section{Corresponding author}

\section{Clancy J. Clark, MD}

Division of Surgical Oncology

Department of General Surgery

Medical Center Blvd.

Winston-Salem, North Carolina 27157

Fax: +1-336-716-9758

cjclark@wakehealth.edu
Background and study aims: In the elderly population, there is a growing demand for minimally invasive procedures as the incidence of pancreaticobiliary disease increases with age. Patients with advanced age offer unique challenges for any procedure because they also tend to have a higher rate of baseline comorbidities and malignancy. The aim of the current study was to characterize the mortality and length of stay of octogenarians undergoing inpatient endoscopic retrograde cholangiopancreatography (ERCP).

Patients and methods: Using the 2007-2010 Nationwide Inpatient Sample (NIS), we performed a retrospective analysis of health-related outcomes among 80- to 89-year-old patients undergoing inpatient ERCP. Surgical patients were excluded. Results: An estimated 61,322 octogenarians underwent inpatient ERCP in the United States from 2007 to 2010. The mean age was 84.2 (SE 0.02)

\section{Introduction}

The growing elderly population in the United States has led to escalating healthcare utilization and expenditures. Healthcare expenses associated with the baby boomer generation grew $7.6 \%$ from 2002 to 2010, which was faster than in any other age group [1]. Concomitantly, the demand for ERCP in the elderly has risen secondary to a high incidence of pancreaticobiliary disease in this population [2-3]. By the time a patient turns 70 , the prevalence of gallbladder-related disease in the United States is 33\% for females and 25\% for males [4].

ERCP is now widely available in the United States at both academic and local non-teaching hospitals. Known complications from ERCP include acute pancreatitis, perforation, infection, gastrointestinal bleeding, adverse effect of anesthetic agents, and death [3]. There are few studies available regarding the safety of ERCP in patient aged 80 years and older [ $5-9$ ]. These small, single-in- with $59.5 \%(n=36,460)$ of the patients being female. A large majority of the patients were white (79. $\%, n=41,144)$ and $63.5 \%(n=38,940)$ had a comorbidity index of at least 2. The mean length of stay was 7.1 days (SE 0.08) with an in-hospital mortality of $3.1 \%(n=1,919)$. The primary discharge diagnosis was most often biliary stone disease $(55.9 \%, n=34,263)$. A diagnosis of any infection was recorded in $45.0 \%(n=27,609)$ of patients. Infection was associated with a significantly higher risk of in-hospital mortality (OR 3.3, 95\% CI 2.6-4.2, $P<0.001$ ).

Conclusions: ERCP is now routinely being performed during inpatient admissions for octogenarians with diseases of the biliary tract. The mortality of octogenarians undergoing inpatient ERCP is higher than previous reports and is likely due to superimposed infection during the same admission.

stitution studies suggest that ERCP is safe in the elderly population [10].

The aim of this population-based study is to characterize the mortality and length of stay in octogenarians undergoing inpatient ERCP. To our knowledge, no prior studies have evaluated the in-hospital mortality associated with ERCP in a large cohort of elderly patients. Based on the limited data available, we hypothesize that ERCP is safe to perform on hospitalized octogenarians.

\section{Patients and methods $\nabla$}

\section{Design}

We performed a retrospective analysis of a national inpatient administrative database.

\section{Database}

Our institutional reviewed board approved this study using the Nationwide Inpatient Sample (NIS) which is part of a family of databases devel- 


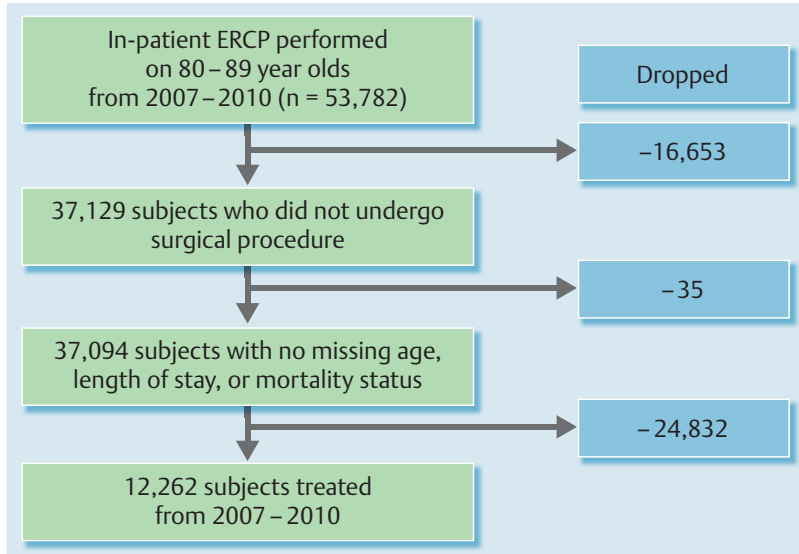

Fig. 1 Cohort selection process represented as raw counts, not United States population estimates

oped and compiled by the Healthcare Cost and Utilization Project (H-CUP) [Agency for Healthcare Research and Quality/H-CUP (December 2014). http:www.hcup-us.ahrq.gov/nisoverview.jsp (February 012015 )]. The NIS is the largest publicly available allpayer inpatient healthcare database in the United States, yielding estimates of hospital inpatient stays. The NIS approximates a $20 \%$ stratified sample of discharges from US community hospitals, which then allows for national-level estimates by using a weighting coefficient provided by the database [10]. Rehabilitation and long-term acute care hospitals are excluded from the database. Policymakers, insurers, and researchers use NIS data to make national estimates of health care utilization, charges, quality, outcomes, and access. The NIS includes data typically found in discharge abstracts, such as up to 25 diagnosis and procedure codes, patient characteristics, length of stay, and payer source. The NIS data excludes individual identifiers.

\section{Sample selection}

We queried the NIS database for all ERCP procedures performed for any indication from 2007 to 2010 on inpatients whose age was 80 to 89 . Admissions that contained complete age, in-hospital mortality data, and length of stay were included in the sample cohort. Patients who also underwent a surgical procedure, such as cholecystectomy, during the same admission were excluded from our study. $\odot$ Fig. 1 outlines the cohort selection process represented as raw counts, not United States population estimates.

\section{Study variables}

Primary outcomes considered in our study were in-hospital mortality and length of stay in octogenarians who underwent ERCP in the United States from 2007 through 2010. In-hospital mortality only includes deaths during the same admission as the ERCP. These mortality data do not include any deaths post-hospitalization. Length of stay was defined as the number of days from admission to the date of discharge. ERCP procedure codes were defined according to the International Classification of Disease, ninth revision, Clinical Modification (ICD-9-CM) system. Therapeutic ERCP procedure codes included both endoscopic operations on biliary ducts and the sphincter of Oddi $(51.84-5.88)$ as well as endoscopic interventions on the pancreas (51.93, 51.94, 51.97, and 51.98). Diagnostic ERCP procedure codes were as follows: ERC (51.11), ERP (52.13), ERCP (51.10), ERCP with biopsy $(51.14,52.14)$, ERCP with excision/destruction of a lesion (51.64,
Table 1 Characteristics of octogenarians who underwent ERCP while hospitalized from 2007 to $2010(n=61,322)$.

\begin{tabular}{|c|c|}
\hline Variable & Estimated \\
\hline Sex, female, n (\%) & $36,460(59.5)$ \\
\hline Age, years, mean (SE) & $84(0.02)$ \\
\hline \multicolumn{2}{|l|}{ Race, $n(\%)^{1}$} \\
\hline White & $41,144(79.9)$ \\
\hline Hispanic & $4,032(7.8)$ \\
\hline African-American & $2,670(5.2)$ \\
\hline Other & $3,669(7.1)$ \\
\hline More than 2 comorbidities, $\mathrm{n}(\%)^{2}$ & $38,940(63.5)$ \\
\hline Alcohol abuse, $\mathrm{n}(\%)$ & $534(0.9)$ \\
\hline \multicolumn{2}{|l|}{ Study period, $n(\%)$} \\
\hline 2007 & $14,996(24.5)$ \\
\hline 2008 & $15,632(25.5)$ \\
\hline 2009 & $15,220(24.8)$ \\
\hline 2010 & $15,473(25.2)$ \\
\hline \multicolumn{2}{|l|}{ Hospital region, $\mathrm{n}(\%)$} \\
\hline Northeast & $15,567(25.4)$ \\
\hline Midwest & $14,539(23.7)$ \\
\hline South & $19,140(31.2)$ \\
\hline West & $12,076(19.7)$ \\
\hline Urban hospital, n (\%) & $56,889(93.1)$ \\
\hline Hospital teaching, $n$ (\%) & $30,188(49.4)$ \\
\hline \multicolumn{2}{|l|}{ Primary payer, $\mathrm{n}(\%)^{3}$} \\
\hline Medicare & $56,182(91.7)$ \\
\hline Medicaid & $973(1.6)$ \\
\hline Private insurance & $3,376(5.5)$ \\
\hline Self-pay, no charge, other & $759(1.2)$ \\
\hline \multicolumn{2}{|l|}{ Admission source, n (\%) } \\
\hline Routine & $6,814(26.3)$ \\
\hline Emergency department & $16,937(65.5)$ \\
\hline Other facility ${ }^{4}$ & $2,123(8.2)$ \\
\hline \multicolumn{2}{|l|}{ Admission type, $\mathrm{n}(\%)$} \\
\hline Emergency & $34,600(64.5)$ \\
\hline Urgent & $12,284(22.9)$ \\
\hline Elective & $6,700(12.5)$ \\
\hline Other & $39(0.07)$ \\
\hline \multicolumn{2}{|l|}{ Hospital bed size, $n(\%)$} \\
\hline Small & $5,366(8.8)$ \\
\hline Medium & $14,331(23.5)$ \\
\hline Large & $41,382(67.8)$ \\
\hline Length of stay, days, mean (SE) & $7.1(0.08)$ \\
\hline In-hospital mortality, n (\%) & $1,919(3.13)$ \\
\hline
\end{tabular}

52.21), and ERCP with manometry (51.15). All of the aforementioned procedure codes were combined to represent the total number of ERCP procedures. Discharge diagnoses were categorized as pancreatitis, stone disease, infection, malignancy, and other (Supplementary $\bullet$ Table 1 ).

Characteristics for patients and hospitals were then extracted from the dataset such as sex, race, age, primary payer, hospital region, teaching status of hospital, hospital size, urban or rural institution, year of admission, source of admission, and number of comorbidities. Hospital size is defined by the NIS and is specific to region, location, and teaching status; for example, a large urban teaching hospital in the south has more than 450 beds [Agency for Healthcare Research and Quality/H-CUP (December 2014). http://www.hcup-us.ahrq.gov/nisoverview.jsp (February 01 2015)]. Each patient was then assigned a comorbidity score 
Table 2 Most common discharge diagnoses for octogenarians undergoing Inpatient ERCP.

\begin{tabular}{|ll|}
\hline & Estimated \\
\hline Discharge diagnosis $^{1}$ & N (\%) \\
\hline Pancreaticobiliary stone disease & $34,263(55.9)$ \\
\hline Malignancy & $18,272(29.8)$ \\
\hline Infection & $27,609(45.0)$ \\
\hline Pancreatitis & $5,765(9.4)$ \\
\hline
\end{tabular}

${ }^{1}$ See Appendix $\bullet$ Supplemental Table 1 for ICD-9 codes.

based on the Elixhauser comorbidity algorithm using validated H-CUP comorbidity software [Agency for Healthcare Research and Quality/H-CUP (October 2011). http://www.hcup-us.ahrq. gov/toolssoftware/comorbidity.jsp (May 01 2015), [11 - 12].

\section{Statistical Analysis}

All analysis was performed in SAS Version 9.4 (Cary, NC). Because NIS implements a stratified, weighted, sampling strategy, all data are presented as national estimates. Appropriate SAS procedures, such as PROC SURVEYMEANS, PROC SURVEYFREQ and PROC SURVEYLOGISTIC, were used to accurately incorporate the sampling strategy of NIS into national estimates. Continuous variables were summarized using means and standard errors, and nominal variables were summarized using counts and percentages. To determine characteristics associated with mortality, univariate and multi-variable logistic regression were used. Variables with overall p-values less than 0.10 were retained in the multi-variable model.

\section{Results}

$\nabla$

An estimated 61,322 octogenarians underwent inpatient ERCP in the United States from 2007 to 2010 . The baseline characteristics of the patients and hospitals are shown in 0 Table 1 . The mean age at time of admission was 84.2 (SE 0.02) with $59.5 \%(n=$ 36,460 ) of the patients being female. A large majority of the patients were white $(79 . \%, n=41,144)$ and $63.5 \%(n=38,940)$ had a comorbidity index of at least 2.ERCP was usually performed at large $(67.7 \%, n=41,382)$, urban $(93.1 \%, n=56,889)$ institutions. The procedures were equally distributed between teaching (50.6\%) and non-teaching hospitals (49.4\%). Most of the admissions were classified as emergent $(64.5 \%, \mathrm{n}=34,600)$. Medicare was the leading payer source $(91.7 \%, n=56,182)$.

The mean length of stay (LOS) was 7.1 days (SE 0.08). The most common discharge diagnosis was pancreaticobiliary stone disease in 55.9\% ( $\bullet$ Table 2 ). The overall in-hospital mortality for octogenarians who had an ERCP performed during the admission was $3.1 \%(n=1,919)$. Risk factors for in-hospital mortality were evaluated. Odds ratio (OR) estimates were calculated for the variables listed in Table 3. Any infection (OR 3.3, 95\% CI 2.6-4.2, $P<0.001$ ), presence of more than 2 comorbidities (OR 2.4, 95\% CI 1.9-3.2, $P<0.001$ ), and malignancy (OR 1.6, 95\%CI 1.6-2.0, $P<0.001$ ) were all associated with higher risk of in-hospital mortality. Age, sex, ethnicity, and hospital characteristics (size, region, rural or urban) were not associated with increased mortality. In multivariate analysis, octogenarians with any infection had a significantly higher risk of in-hospital mortality (OR 3.1, 95\% CI $2.5-4.0, P<0.001$ ). The presence of more than 2 more comorbidities, malignancy, and increasing age were also associated with slightly higher in-hospital mortality in the multivariate analysis.

Table 3 Risk factors for in-hospital mortality in octogenarians undergoing ERCP

\begin{tabular}{|c|c|c|c|c|c|c|c|c|}
\hline \multirow[b]{2}{*}{ Variable } & \multicolumn{4}{|c|}{ Univariate analysis } & \multicolumn{4}{|c|}{ Multiple variable analysis } \\
\hline & OR & $95 \%$ CI Lower & 95\% CI Upper & p-value & OR & 95\% CI Lower & 95\% CI Upper & $P$ value \\
\hline $\mathrm{Age}^{1}$ & 1.05 & 0.997 & 1.096 & 0.0674 & 1.050 & 1.001 & 1.101 & 0.0463 \\
\hline Male sex & 1.207 & 0.984 & 1.481 & 0.0717 & 0.870 & 0.706 & 1.071 & 0.1879 \\
\hline More than 2 comorbidities ${ }^{2}$ & 2.447 & 1.868 & 3.206 & $<0.001$ & 1.446 & 1.285 & 1.626 & $<0.001$ \\
\hline Race, non-white & 1.174 & 0.911 & 1.513 & 0.2145 & & & & \\
\hline \multicolumn{9}{|l|}{ Hospital size } \\
\hline Small & ref & & & & & & & \\
\hline Medium & 1.162 & 0.731 & 1.845 & 1.162 & & & & \\
\hline Large & 1.218 & 0.791 & 1.874 & 0.426 & & & & \\
\hline Non-teaching hospital & 0.772 & 0.631 & 0.944 & 0.0117 & 0.787 & 0.641 & 0.966 & 0.0218 \\
\hline \multicolumn{9}{|l|}{ Region } \\
\hline West & ref & & & & & & & \\
\hline Northeast & 0.993 & 0.736 & 1.340 & 0.9646 & & & & \\
\hline Midwest & 0.825 & 0.611 & 1.114 & 0.2086 & & & & \\
\hline South & 0.942 & 0.720 & 1.232 & 0.6618 & & & & \\
\hline Rural hospital & 1.002 & 0.706 & 1.421 & 0.9932 & & & & \\
\hline $\begin{array}{l}\text { Admission source, } \\
\text { emergency department }\end{array}$ & 0.954 & 0.669 & 1.361 & 0.7952 & & & & \\
\hline \multicolumn{9}{|l|}{ Admission type } \\
\hline Urgent or emergent & ref & & & & ref & & & \\
\hline Elective & 0.642 & 0.423 & 0.976 & 0.038 & 0.748 & 0.483 & 1.157 & 0.1919 \\
\hline Any stone disease & 0.533 & 0.433 & 0.656 & $<0.001$ & 0.571 & 0.441 & 0.739 & $<0.001$ \\
\hline Any infection & 3.329 & 2.649 & 4.184 & $<0.001$ & 3.105 & 2.523 & 4.070 & $<0.001$ \\
\hline Any malignancy & 1.616 & 1.320 & 1.977 & $<0.001$ & 1.28 & 1.005 & 1.629 & 0.00453 \\
\hline Any pancreatitis & 0.751 & 0.571 & 0.988 & 0.0405 & 0.960 & 0.723 & 1.274 & 0.7771 \\
\hline
\end{tabular}

${ }^{1}$ For each additional year.

2 Defined by Elixhauser comorbidity algorithm $[13,14]$ 


\section{Discussion}

\section{$\nabla$}

Our study determined that the in-hospital mortality for octogenarians who undergo inpatient ERCP is $3.1 \%$. To our knowledge, this is the first large-scale population-based study to examine mortality and length of stay in octogenarians undergoing inpatient ERCP. Because this analysis used a nationally representative sample of octogenarian patients who underwent inpatient ERCP in the community setting, these findings can be generalized to the overall US octogenarian population.

Prior reports would suggest that ERCP is safe and without increased mortality or complication rates in octogenarians [3, 59]. In one retrospective review comparing 102 patients older than age 80 to a younger cohort, ERCP was both safe and efficacious without an increase in complication rates [5]. Although ERCP procedure time was usually longer and the case technically more challenging in octogenarians, ERCP was not found to have higher complication rates in this elderly cohort [6]. In fact, rates of post-ERCP infection and pancreatitis were lower in octogenarians.

Generally, all patients who undergo ERCP procedures have a mortality of less than $0.5 \%$ [13-15]. Prior small studies focusing on the very elderly such as octogenarians found a mortality rate of $0-1 \%[3,6-10,16]$. The mortality of octogenarians in our study was over $3 \%$. Almost half of the hospitalized octogenarians we evaluated had an infection during the same hospitalization at the time of ERCP and one-third had a diagnosed malignancy. Infection and malignancy includes both biliary and non-biliary etiologies such as pneumonia and lymphoma. Given concern for selection bias using an administrative dataset, we did not perform a subset analysis of patients diagnosed with cholangitis. However, cholangitis is likely a significant factor in increased inhospital morbidity and mortality. Many octogenarians also had numerous comorbidities and the presence of more than 2 comorbidities was associated with higher inpatient mortality.

Accordingly, the finding of higher mortality in octogenarians is best explained by the increased rates of infections and malignancy in this population with baseline comorbidities. Although, the mortality rate in this study for octogenarians undergoing inpatient ERCP is higher than in previous reports, the procedure remains necessary and efficacious for many individuals. With careful patient selection and high-quality periprocedural care, we can likely minimize risks associated with ERCP in octogenarians. We recommend that patients and families undergo appropriate informed consent including information on inpatient mortality before any inpatient ERCP.

The average length of stay for octogenarians undergoing ERCP was 7.1 days in our study. This is comparable to the reported length of stay for all age groups undergoing inpatient ERCP of $6.9-7.8$ days [15]. Our large sample size should mitigate the effects of individual hospital characteristics on length of stay such as a lower procedure volume or fewer critical care resources.

Our study has a few limitations. The NIS database allows for only the assessment of inpatient mortality, morbidity, and length of stay. Death after discharge or complications requiring readmission cannot be tracked or evaluated because the NIS data are only from inpatient hospitalizations and contains no individual identifiers. We cannot comment on the mortality in outpatient octogenarians who undergo ERCP as this study only utilized hospitalized patients. All patients who underwent any surgical procedure during the same admission were excluded from the analysis in an attempt to limit confounders on inpatient mortality in this very elderly cohort. By excluding surgical patients, we may be eliminating some healthier patients that were surgical candidates and thus potentially would have lowered the mortality. Conversely, we are also excluding patients that had potential ERCP morbidities such as perforation that would have required urgent surgery and likely had a higher mortality.

\section{Conclusion \\ $\nabla$}

In this large-scale population-based study of inpatient octogenarians undergoing ERCP, the rate of in-hospital mortality was $3.1 \%$ and the length of stay was 7.1 days. Although the length of stay was comparable to all age groups, the mortality was higher than in previous reports and is attributable to concomitant infection and malignancy in this elderly cohort with baseline comorbidities.

\section{Competing interests: None}

\section{References}

1 Lasman D, Hartman M, Washington B et al. US Health spending trends by age and gender: selected years $2002-2010$. Health Affairs 2014; 33 : 815-822 DOI 10.1377/hlthaff.2013.1224

2 Harness JK, Strodel WE, Talsma SE. Symptomatic biliary tract disease in the elderly patient. The American Surgeon 1986; 56: 586-590

3 Behlül B, Ayfer S, Vatansever $S$ et al. Safety of endoscopic retrograde cholangiopancreatography in patients 80 years of age and older. Przeglad Gastroenterologiczny 2014; 9: 227-231

4 Everhart JE, Khare M, Hill $M$ et al. Prevalence and ethnic differences in gallbladder disease in the United States. Gastroenterology 1999; 117: $632-639$

5 Ali $M$, Ward G, Staley $D$ et al. A retrospective study of the safety and efficacy of ERCP in octogenarians. Digestive Diseases and Sciences 2011; 56: $586-590$

6 Lukens FJ, Howell DA, Upender $S$ et al. ERCP in the very elderly; outcomes among patients older than eighty. Dig Dis Sci 2010; 55: 847 851

7 Fritz E, Kirchgatterer A, Hubner D et al. ERCP is safe and effective in patients 80 years of age and older compared with younger patients. Gastrointest Endosc 2006; 64: 899-905

$8 \mathrm{Kim}$ JE, Cha BH, Lee SH et al. Safety and efficacy of endoscopic retrograde cholangiopancreatography in very elderly patients. Korean J Gastroenterol 2011; 57: 237-242

9 Cho DH, Park GT, Oh JE et al. A single institution's experience of endoscopic retrograde cholangiopancreaticography in the elderly patients: outcomes, safety, and complications. Korean J Gastroenterol 2011; 58: $88-92$

10 Katsinelos $P$, Kountouras J, Chatzimavroudis $G$ et al. Outpatient therapeutic endoscopic retrograde cholangiopancreatography is safe in patients aged 80 years and older. Endoscopy 2011; 43: 128-133 DOI 10.1055/s-0030-1255934

11 Elixhauser A, Steiner C, Harris DR et al. Comorbidity measures for use with administrative data. Med Care 1998; 36: 8-27

12 Grendar J, Shaheen AA, Myers RP et al. Predicting in-hospital mortality in patients undergoing complex gastrointestinal surgery: determining the optimal risk adjustment method. Arch Surg 2012; 147: 126-135

13 Varadarajulu S, Kilgore ML, Wilcox CM et al. Relationship among hospital ERCP volume, length of stay, and technical outcomes. Gastrointest Endosc 2006; 64: 338 - 347

14 Masci E, Toti G, Mariani A et al. Complications of diagnostic and therapeutic ERCP: a prospective multicenter study. Am J Gastroenterol 2001; 96: $417-423$

15 Vandervoort J, Soetikno RM, Tham TC et al. Risk factors for complications after performance of ERCP. Gastrointest Endosc 2002; 56: 652 656

16 Yun DY, Han J, Oh JS et al. Is endoscopic retrograde cholangiopancreatography safe ub oatuebts 90 years of age and older? Gut Liver 2014; 8: $552-556$ 


\section{Supplemental Table 1 ICD-9 codes for discharge diagnosis categories.}

\section{Discharge diagno- ICD-9 codes}

sis category

Stone disease

560.31-gallstone ileus, 574.00-claculus of gallbladder with acute cholecystitis without obstruction, 574.01 - claculus of gallbladder with acute cholecystitis with obstruction, 574.10-calculus of gallbladder with other cholecystitis without obstruction, 574.11-calculus of gallbladder with other cholecystitis with obstruction, 574.20-calculus of gallbladder without cholecystitis without obstruction, 574.21-calculus of gallbladder without cholecystitis with obstruction, 574.30-calculus of bile duct with acute cholecystitis without obstruction, 574.31-calculus of bile duct with acute cholecystitis with obstruction, , 574.40-calculus of bile duct with other cholecystitis without obstruction, 574.41-calculus of bile duct with other cholecystitis with obstruction,, 574.50-calculus of bile duct without cholecystitis without obstruction, 574.51 calculus of bile duct without cholecystitis with obstruction, 574.60-calculus of gallbladder and bile duct with acute cholecystitis without obstruction, 574.61-calculus of gallbladder and bile duct with acute cholecystitis with obstruction, 574.70-calculus of gallbladder and bile duct with other cholecystitis without obstruction, 574.71-calculus of gallbladder and bile duct with other cholecystitis without obstruction, 574.80 -calculus of gallbladder and bile duct with acute and chronic cholecystitis without obstruction, 574.81-calculus of gallbladder and bile duct with acute and chronic cholecystitis with obstruction, 574.90-calculus of gallbladder and bile duct without cholecystitis without obstruction, 574.91-calculus of gallbladder and bile duct without cholecystitis with obstruction, 997.4-retained cholelithiasis following cholecystectomy 
Supplemental Table 1 (Continuation)

\section{Discharge diagno- ICD-9 codes sis category}

Malignancy

147.1-malignant neoplasm of posterior wall of nasopharynx, 147.9-malignant neoplasm of nasopharynx unspecified site, 150.5malignant neoplasm of lower third of esophagus, 150.8-malignant neoplasm of other specified part of esophagus, 151.0-malignant neoplasm of cardia, 151.1-malignant neoplasm of pylorus, 151.2-malignant neoplasm of pyloric antrum, 151.3-malignant neoplasm of fundus of stomach, 151.4-malignant neoplasm of body of stomach, 151.5-malignant neoplasm of lesser curvature of stomach unspecified, 151.6-malignant neoplasm of greater curvature of stomach unspecified, 151.8-malignant neoplasm of other specified sites of stomach, 151.9-malignant neoplasm of stomach unspecified site, 152.0-malignant neoplasm of duodenum, 152.1malignant neoplasm of jejunum, 152.2-malignant neoplasm of ileum, 152.8-malignant neoplasm of other specified sites of small intestine, 152.9-malignant neoplasm of small intestine unspecified site, 153.0-malignant neoplasm of hepatic flexure, 153.1-malignant neoplasm of transverse colon, 153.2-malignant neoplasm of descending colon, 153.3-malignant neoplasm of sigmoid colon, 153.4-malignant neoplasm of cecum, 153.6-malignant neoplasm of ascending colon, 153.7-malignant neoplasm of splenic flexure, 153.8-malignant neoplasm of other specified sites of large intestine, 153.9-malignant neoplasm of colon unspecified site, 154.0malignant neoplasm of rectosigmoid junction, 154.1-malignant neoplasm of rectum, 154.3-malignant neoplasm of anus unspecified site, 154.8-malignant neoplasm of other sites of rectum, rectosigmoid junction, and anus, 155.0-malignant neoplasm of liver primary, 155.1-malignant neoplasm of intrahepatic bile ducts, 155.2-malignant neoplasm of liver not specified as primary or secondary, 156.0-malignant neoplasm of gallbladder, 156.1-malignant neoplasm of extrahepatic bile ducts, 156.2-malignant neoplasm of ampulla of vater, 156.8-malignant neoplasm of other specified sites of gallbladder and extrahepatic bile ducts, 156.9-malignant neoplasm of biliary tract part unspecified site, 157.0-malignant neoplasm of head of pancreas, 157.1-malignant neoplasm of body of pancreas, 157.2-malignant neoplasm of tail of pancreas, 157.3-malignant neoplasm of pancreatic duct, 157.4-malignant neoplasm of islets of Langerhans, 157.8-malignant neoplasm of other specified sites of pancreas, 157.9-malignant neoplasm of pancreas part unspecified, 158.0-malignant neoplasm of retroperitoneum, 158.8-malignant neoplasm of specified parts of peritoneum, 158.9-malignant neoplasm of peritoneum unspecified, 159.0-malignant neoplasm of intestinal tract part unspecified, 159.8malignant neoplasm of other sites of digestive system and intra-abdominal organs, 162.2-malignant neoplasm of main bronchus, 162.3-malignant neoplasm of upper lobe bronchus or lung, 162.5-malignant neoplasm of lower lobe bronchus or lung, 162.8-malignant neoplasm of other parts of bronchus or lung, 162.9-malignant neoplasm of bronchus and lung unspecified, 170.6-malignant neoplasm of pelvic bones sacrum and coccyx, 173.3-unspecified malignant neoplasm of skin of other and unspecified parts of face, 173.4-unspecified malignant neoplasm of scalp and skin of neck, 174.8-malignant neoplasm of other specified sites of female breast, 174.9-malignant neoplasm of breast (female) unspecified site, 182.0-malignant neoplasm of corpus uteri except isthmus, 183.0-malignant neoplasm of ovary, 185-malignant neoplasm of prostate, 188.2-malignant neoplasm of lateral wall of urinary bladder, 188.8-malignant neoplasm of other specified sites of bladder, 188.9-malignant neoplasm of bladder part unspecified, 189.0-malignant neoplasm of kidney except pelvis, 189.2-malignant neoplasm of ureter, 194.0-malignant neoplasm of adrenal gland, 195.0-malignant neoplasm of head face and neck, 195.2-malignant neoplasm of abdomen, 195.8-malignant neoplasm of other specified sites, 196.2-secondary and unspecified malignant neoplasm of intra-abdominal lymph nodes, 196.3-secondary and unspecified malignant neoplasm of lymph nodes of axilla and upper limb, 197.0-secondary malignant neoplasm of lung, 197.1-secondary malignant neoplasm of mediastinum, 197.2-secondary malignant neoplasm of pleura, 197.4-secondary malignant neoplasm of small intestine including duodenum, 197.5-secondary malignant neoplasm of large intestine and rectum, 197.6-secondary malignant neoplasm of retroperitoneum and peritoneum, 197.7-malignant neoplasm of liver secondary, 197.8 -secondary malignant neoplasm of other digestive organs and spleen, 198.1-secondary malignant neoplasm of other urinary organs, 198.2-secondary malignant neoplasm of skin, 198.3-secondary malignant neoplasm of brain and spinal cord, 198.5-secondary malignant neoplasm of bone and bone marrow, 198.7-secondary malignant neoplasm of adrenal gland, 198.89-secondary malignant neoplasm of other specified sites, 199.0-disseminated malignant neoplasm, 199.1-other malignant neoplasm of unspecified site, 200.00-reticulosarcoma unspecified site, 200.02-reticulosarcoma involving intrathoracic lymph nodes, 200.03-reticulosarcoma involving intra-abdominal lymph nodes, 200.07-reticulosarcoma involving spleen, 200.08-reticulosarcoma involving lymph nodes of multiple sites, 200.23-Burkitt's tumor or lymphoma involving intra-abdominal lymph nodes, 200.40-mantle cell lymphoma, unspecified site, extranodal and solid organ sites, 200.70-large cell lymphoma, unspecified site, extranodal and solid organ sites, 200.71-large cell lymphoma, lymph nodes of head, face, and neck, 200.80-other named variants of lymphosarcoma and reticulosarcoma unspecified site, 201.50-Hodgkin's disease nodular sclerosis unspecified site, 201.55-Hodgkin's disease nodular sclerosis involving lymph nodes of inguinal region and lower limb, 201.90-Hodgkin's disease unspecified type unspecified site, 202.03-Nodular lymphomas involving intra-abdominal lymph nodes, 202.80-other malignant lymphomas unspecified site, 202.83 - other malignant lymphomas involving intra-abdominal lymph nodes, 202.84 other malignant lymphomas involving lymph nodes of axilla and upper limb, 202.85other malignant lymphomas involving lymph nodes of ingoing region and lower limb, 202.86 - other malignant lymphomas involving intrapelvic lymph nodes, 202.88 - other malignant lymphomas involving lymph nodes of multiple sites, 203.00-multiple myeloma without mention of having achieved remission, 204.00-acute lymphoid leukemia without mention of having achieved remission, 204.10-chronic lymphoid leukemia without mention of having achieved remission, 205.00-acute myeloid leukemia without mention of having achieved remission, 205.10-chronic myeloid leukemia without mention of having achieved remission, 209.00malignant carcinoid tumor of the small intestine, unspecified portion, 209.23-malignant carcinoid tumor of the stomach, 209.72secondary neuroendocrine tumor of liver, 211.1-benign neoplasm of stomach, 211.2-benign neoplasm of duodenum jejunum and ileum, 211.3-benign neoplasm of colon, 211.5-benign neoplasm of liver and biliary passages, 211.6-benign neoplasm of pancreas except islets of Langerhans, 215.0-other benign neoplasm of connective and other soft tissue of head face and neck, 220-benign neoplasm of ovary, 225.2-benign neoplasm of cerebral meninges, 227.1-benign neoplasm of parathyroid gland, 230.2-carcinoma in situ of the stomach, 230.7-carcinoma in situ of other and unspecified parts of intestine, 230.8-carcinoma in situ of liver and biliary system, 230.9-carcinoma in situ of other and unspecified digestive organs, 235.2-neoplasm of uncertain behavior of stomach intestines and rectum, 235.3-neoplasm of uncertain behavior of liver and biliary passages, 235.5-neoplasm of uncertain behavior of other and unspecified digestive organs, 237.5-neoplasm of uncertain behavior of brain and spinal cord, 238.73-high grade myelodysplastic syndrome lesions, 239.0-neoplasm of unspecified nature of digestive system, 239.5-neoplasm of unspecified nature of other genitourinary organs, 239.89-neoplasms of unspecified nature other specified sites, v58.11-encounter for antineoplastic chemotherapy, v71.1-observation for suspected malignant neoplasm 


\begin{tabular}{|c|c|}
\hline \multicolumn{2}{|c|}{ Supplemental Table $1 \quad$ (Continuation) } \\
\hline $\begin{array}{l}\text { Discharge diagno- } \\
\text { sis category }\end{array}$ & ICD-9 codes \\
\hline Infection & 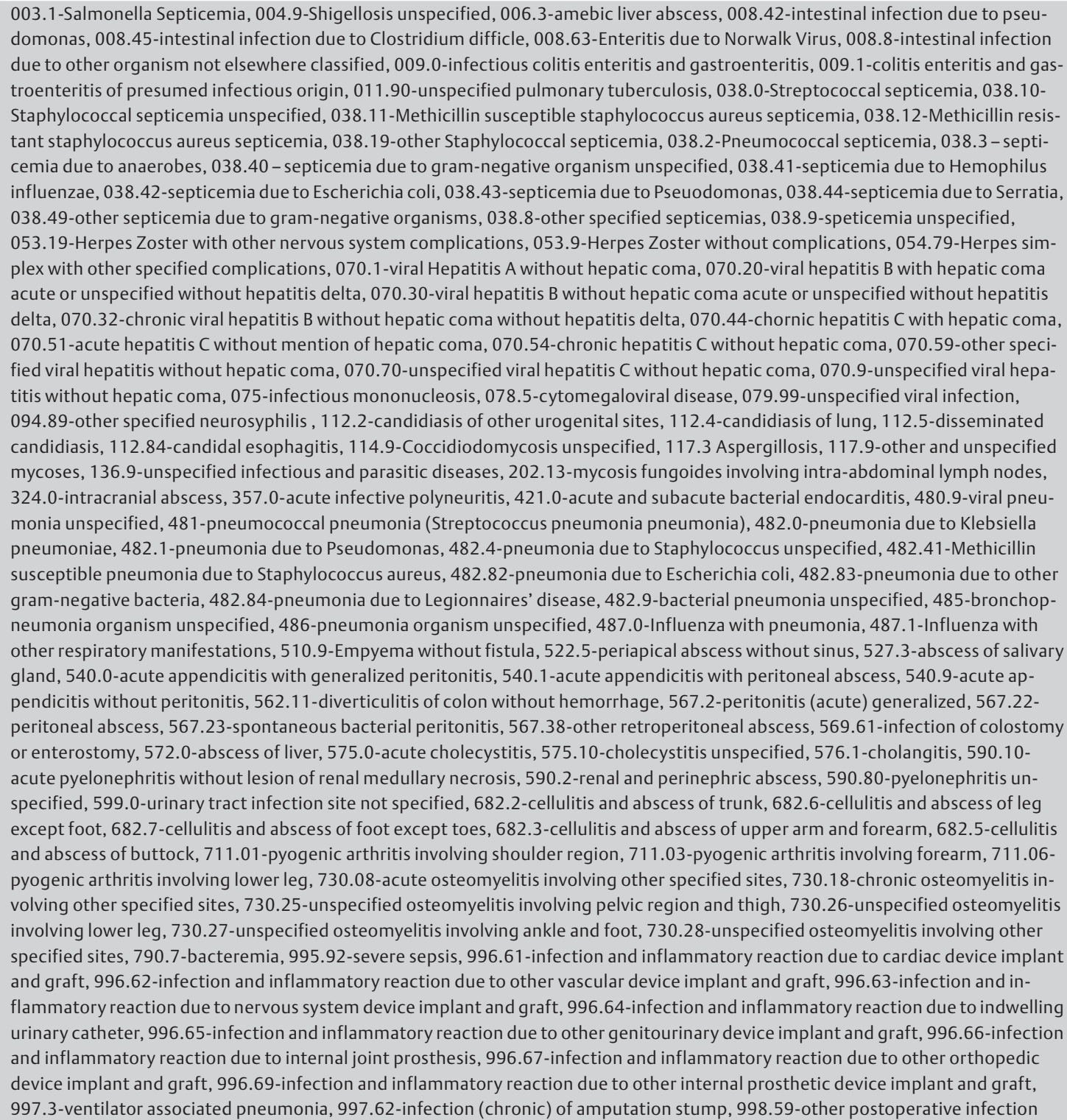 \\
\hline Pancreatitis & 577.0-acute pancreatitis, 577.1-chronic pancreatitis \\
\hline Other & All remaining primary discharge codes. \\
\hline
\end{tabular}

